\title{
APPROXIMATING SET MULTI-COVERS
}

\author{
MÁRTON NASZÓDI AND ALEXANDR POLYANSKII
}

\begin{abstract}
Johnson and Lovász and Stein proved independently that any hypergraph satisfies $\tau \leq(1+\ln \Delta) \tau^{*}$, where $\tau$ is the transversal number, $\tau^{*}$ is its fractional version, and $\Delta$ denotes the maximum degree. We prove $\tau_{f} \leq 3.153 \tau^{*} \max \{\ln \Delta, f\}$ for the $f$-fold transversal number $\tau_{f}$. Similarly to Johnson, Lovász and Stein, we also show that this bound can be achieved non-probabilistically, using a greedy algorithm.

As a combinatorial application, we prove an estimate on how fast $\tau_{f} / f$ converges to $\tau^{*}$. As a geometric application, we obtain an upper bound on the minimal density of an $f$-fold covering of the $d$-dimensional Euclidean space by translates of any convex body.
\end{abstract}

\section{Introduction and Preliminaries}

A hypergraph is a pair $(X, \mathcal{F})$, where $X$ is a finite set and $\mathcal{F} \subseteq 2^{X}$ is a family of some subsets of $X$. We call the elements of $X$ vertices, and the members of $\mathcal{F}$ edges of the hypergraph. When a vertex is contained in an edge, we may say that 'the vertex covers the edge', or that 'the edge covers the vertex'.

Let $f$ be a positive integer. An $f$-fold transversal of $(X, \mathcal{F})$ is a multiset $A$ of $X$ such that each member of $\mathcal{F}$ contains at least $f$ elements (with multiplicity). The $f$-fold transversal number $\tau_{f}$ of $(X, \mathcal{F})$ is the minimum cardinality (with multiplicity) of an $f$-fold transversal. A 1-transversal is called a transversal, and the 1-transversal number is called the transversal number, and is denoted by $\tau=\tau_{1}$.

A fractional transversal is a function $w: X \longrightarrow[0,1]$ with $\sum_{x: x \in F} w(x) \geq 1$ for all $F \in \mathcal{F}$. The fractional transversal number of $(X, \mathcal{F})$ is

$$
\tau^{*}=\tau^{*}(\mathcal{F}):=\inf \left\{\sum_{x: x \in X} w(x): w \text { is a fractional transversal }\right\} .
$$

Clearly, $\tau^{*} \leq \tau$. In the opposite direction, Johnson [Joh74], Lovász [Lov75] and Stein [Ste74 independently proved that

$$
\tau \leq(1+\ln \Delta) \tau^{*},
$$

where $\Delta$ denotes the maximum degree of $(X, \mathcal{F})$, that is, the maximum number of edges a vertex is contained in. They showed that the greedy algorithm, that is, picking vertices of $X$ one by one, in such a way that we always pick one that is contained in the largest number of uncovered edges, yields a transversal set whose cardinality does not exceed the right hand side in (11). For more background, see Füredi's survey [Für88].

Our main result is an extension of this theorem to $f$-fold transversals.

2010 Mathematics Subject Classification. 05D15, 52C17.

Key words and phrases. transversal, covering, Rogers' bound, multiple transversal.

M. Naszódi thanks the following agencies for their support: the Swiss National Science Foundation grants no. 200020-162884 and 200021-175977; the János Bolyai Research Scholarship of the Hungarian Academy of Sciences; and the National Research, Development and Innovation Office, NKFIH Grants PD-104744 and K119670.

A. Polyanskii was partially supported by the Russian Foundation for Basic Research, grants 15-3120403 (mol_a_ved), 15-01-99563 A, 15-01-03530 A. 
Theorem 1.1. Let $\lambda \in(0,1)$ and let $f$ be a positive integer. Then, with the above notation,

$$
\tau_{f} \leq \frac{1-\lambda^{f}}{1-\lambda} \tau^{*}(1+\ln \Delta-(f-1) \ln \lambda),
$$

moreover, for rational $\lambda$, the greedy algorithm using appropriate weights, yields an $f$-fold transversal of cardinality not exceeding the right hand side of (2).

Substituting $\lambda=0.287643$ (which is a bit less than $1 / e$ ), we obtain

Corollary 1.2. With the above notation, we have

$$
\tau_{f} \leq 3.153 \tau^{*} \max \{\ln \Delta, f\} .
$$

This result may be interpreted in two ways. First, it gives an algorithm that approximates the integer programming (IP) problem of finding $\tau_{f}$, with a better bound on the output of the algorithm than the obvious estimate $\tau_{f} \leq f \tau \leq f \tau^{*}(1+\ln \Delta)$.

A similar result was obtained by Rajagopalan and Vazirani in [RV98] (an improvement of [Dob82]), where, the (multi)-set (multi)-cover problem is considered, that is, the goal is to cover vertices by sets. This is simply the combinatorial dual (and therefore, equivalent) formulation of our problem. In [RV98, each set can be chosen at most once. They present generalizations of the greedy algorithm of [Joh74], [Lov75] and [Ste74], and prove that it finds an approximation of the (multi)-set (multi)-cover problem within an $\ln \Delta$ factor of the optimal solution of the corresponding linear programming (LP) problem. Moreover, they give parallelized versions of the algorithms.

The main difference between [RV98, and the present paper is that there, the optimal solution of an IP problem is compared to the optimal solution of the LP-relaxation of the same IP problem, whereas here, we compare $\tau_{f}$ with $\tau^{*}$, where the latter is the optimal solution of a weaker LP problem: the problem with $f=1$.

We note that, using the fact that $f \tau^{*} \leq \tau_{f}$, (3) also implies that the performance ratio (that is, the ratio of the value obtained by the algorithm to the optimal value, in the worst case) of our algorithm is constant when $\ln \Delta \leq f$. Compare this with [BDS04, Lemma 1 in Section 3.1], where it is shown that, even for large $f$, the standard greedy algorithm yields a performance ratio of $\Omega(\ln m)$, where $m$ is the number of sets in the hypergraph. Further recent results on the performance ratio of another modified greedy algorithm for variants of the set cover problem can be found in FK06. See also Chapter 2 of the book Vaz01 by Vazirani.

The second interpretation of our result is the following. It is easy to see that $\frac{\tau_{f}}{f}$ converges to $\tau^{*}$ as $f$ tends to infinity. Now, (3) quantifies the speed of this convergence in some sense. In particular, it yields that for $f=\ln \Delta$ we have $\frac{\tau_{f}}{f} \leq 3.153 \tau^{*}$. We have better approximation for larger $f$.

Corollary 1.3. For every $0<\varepsilon \leq 1$, if we set $f:=\left\lceil\frac{2(1+\ln \Delta)}{\varepsilon(1-\lambda)}\right\rceil$, where $0<\lambda<1$ is such that $-\ln \lambda /(1-\lambda) \leq 1+\varepsilon / 2$, then the $f$-fold transversal constructed in Theorem 1.1 yields a fractional transversal which gives

$$
\tau^{*} \leq \frac{\tau_{f}}{f} \leq \tau^{*}(1+\varepsilon) .
$$

We prove Theorem 1.1 and Corollary 1.3 in Section 2, where, at the end, we discuss the running time of our algorithm. 
1.1. A geometric application. Next, we turn to a classical geometric covering problem. Rogers [Rog57 showed that for any convex body $K$ in $\mathbb{R}^{d}$, there is a covering of $\mathbb{R}^{d}$ with translates of $K$ of density at most

$$
d \ln d+d \ln \ln d+5 d
$$

For the definition of density cf. [PA95]. G. Fejes Tóth [FT76, FT79] gave the non-trivial lower bound $c_{d} f$ for the density of an $f$-fold covering of $\mathbb{R}^{d}$ by Euclidean unit balls (with some $c_{d}>1$ ). For more information on multiple coverings in geometry, see the survey [FT04]. As an application of Theorem 1.1, we give a similar estimate for $f$-fold coverings.

Theorem 1.4. Let $K \subseteq \mathbb{R}^{d}$ be a convex body and $f \geq 1$ an integer. Then there is an arrangement of translates of $K$ with density at most

$$
(1+o(1)) \cdot 3.153 \max \{d \ln d, f\},
$$

where every point of $\mathbb{R}^{d}$ is covered at least $f$ times.

The key in proving Theorem 1.4 is a general statement, Theorem 3.1, presented in Section 3. Both theorems are proved the same way as corresponding results in [Nas14, where the case $f=1$ is considered.

Earlier versions of Theorems 1.4 and 3.1 were proved in [FNN16. There, in place of the main result of the present paper, a probabilistic argument is used which yields quantitatively weaker bounds. The quantitative gain here comes from the fact that in the probabilistic bound on $\tau_{f}$ presented in [FNN16], one has the size of the edge set $\mathcal{F}$ as opposed to the maximum degree $\Delta$, which is what we have in (3).

\section{Proof of Theorem 1.1}

2.1. The algorithm. First, we imagine that each member of $\mathcal{F}$ has $f$ bank notes, the denominations are $\$ 1, \$ \lambda, \ldots, \$ \lambda^{f-1}$, where $\lambda<1$ is fixed. We pick vertices one by one. At each step, we pick a vertex, and each edge that contains it pays the largest bank note that it has. So, each edge pays $\$ 1$ for the first vertex selected from it, then $\$ \lambda$ for the second, etc., and finally, $\$ \lambda^{f-1}$ for the $f$-th vertex that it contains. Later on, it does not pay for any additional selected vertex that it contains. Now, we follow the greedy algorithm: at each step, we pick the vertex that yields the largest payout at that step. We finish once each edge is covered at least $f$ times, that is, when we collected all the money.

2.2. Notation. Given a positive integer $f$, we define the truncated exponential function denoted by ${ }^{k} \lambda$ as follows: for any $\lambda>0$, and any $0 \leq k<f$, let ${ }^{k} \lambda=\lambda^{k}$, and let ${ }^{k} \lambda=0$ for any $k \geq f$. Note that the value of $f$ is implicitly present in any formula involving the truncated exponential function.

For each $F \in \mathcal{F}$, let $k(F)$ denote the number of chosen vertices (with multiplicity) contained in $F$. We call the function $k: \mathcal{F} \rightarrow \mathbb{Z}^{\geq 0}$ the current state, where $\mathbb{Z}^{\geq 0}$ is the set of non-negative integers. At the start, $k$ is identically zero.

Given a function $k: \mathcal{F} \rightarrow \mathbb{Z}^{\geq 0}$, we define the value of a vertex $x \in X$ with respect to $k$ as

$$
v_{k}(x):=\sum_{F: x \in F \in \mathcal{F}}{ }^{k(F)} \lambda .
$$

The total remaining value of $k$ is defined as

$$
v(k):=\sum_{F: F \in \mathcal{F}} \sum_{i=k(F)}^{f}{ }^{i} \lambda,
$$


which is the total pay out that will be earned in the subsequent steps.

2.3. Fractional matchings. A fractional matching of the hypergraph $(X, \mathcal{F})$ is a function $w: \mathcal{F} \longrightarrow[0,1]$ with $\sum_{F: x \in F \in \mathcal{F}} w(F) \leq 1$ for all $x \in X$. The fractional matching number of $(X, \mathcal{F})$ is

$$
\nu^{*}=\nu^{*}(\mathcal{F}):=\sup \left\{\sum_{F: F \in \mathcal{F}} w(F): w \text { is a fractional matching }\right\} .
$$

By the duality of linear programming, $\nu^{*}=\tau^{*}$.

We will need the following simple observation.

Lemma 2.1. Let $z>0$, and $\ell: \mathcal{F} \rightarrow \mathbb{Z}^{\geq 0}$ be such that $v_{\ell}(x) \leq z$ for any $x \in X$. Then we have

$$
\frac{v(\ell)}{z} \leq\left(1+\lambda+\ldots+\lambda^{f-1}\right) \nu^{*}(\mathcal{F})=\frac{1-\lambda^{f}}{1-\lambda} \nu^{*}(\mathcal{F}) .
$$

Proof of Lemma 2.1. Let

$$
w(F):=\frac{\sum_{i=\ell(F)}^{f}{ }^{i} \lambda}{z\left(1+\lambda+\ldots+\lambda^{f-1}\right)}, \text { for any } F \in \mathcal{F} .
$$

First, we show that $w$ is a fractional matching. Indeed, fix an $x \in X$.

Second, the total weight is

$$
\begin{gathered}
\sum_{F: x \in F \in \mathcal{F}} w(F)=\frac{1}{z} \sum_{F: x \in F \in \mathcal{F}} \frac{\sum_{i=\ell(F)}^{f}{ }^{i} \lambda}{1+\lambda+\ldots+\lambda^{f-1}} \leq \\
\leq \frac{1}{z} \sum_{F: x \in F \in \mathcal{F}}{ }^{(F)} \lambda=\frac{v_{\ell}(x)}{z} \leq 1 .
\end{gathered}
$$

$$
\sum_{F: F \in \mathcal{F}} w(F)=\frac{1}{z\left(1+\lambda+\ldots+\lambda^{f-1}\right)} \sum_{F: F \in \mathcal{F}} \sum_{i=\ell(F)}^{f}{ }^{i} \lambda=\frac{v(\ell)}{z\left(1+\lambda+\ldots+\lambda^{f-1}\right)},
$$

finishing the proof of the Lemma.

2.4. Finally, we count the steps of the algorithm. We may assume that $\lambda=p / q \in$ $(0,1)$ with $p, q \in \mathbb{Z}^{+}$. If $\lambda$ is irrational, then the statement of Theorem 1.1 follows by continuity. Clearly, $q^{f-1}$ is a common denominator for the pay outs at each step.

At the start, $k_{0}(F):=k(F)=0$ for all $F \in \mathcal{F}$. We group the steps according to the $\$$-amount (that is, $v_{k}(x)$ ) that we get at each.

In the first $t_{1}$ steps, each vertex $x$ that we pick has value $v_{k}(x)=\Delta=: z_{1}$, where, we recall, $\Delta$ is the maximum degree in the hypergraph. Let $k_{1}: \mathcal{F} \rightarrow \mathbb{Z}^{\geq 0}$ denote the current state after the first $t_{1}$ steps.

Then, in the second group of steps, we make $t_{2}$ steps, at each picking a vertex $x \in V$ of value $v_{k}(x)=\Delta-q^{1-f}=: z_{2}$, where $k$ changes at each step. Let $k_{2}: \mathcal{F} \rightarrow \mathbb{Z}^{\geq 0}$ denote the current state after the first $t_{1}+t_{2}$ steps.

In the $j$-th group of steps, we make $t_{j}$ steps, at each picking a vertex $x \in V$ of value $v_{k}(x)=\Delta-(j-1) q^{1-f}=: z_{j}$. Let $k_{j}: \mathcal{F} \rightarrow \mathbb{Z}^{\geq 0}$ denote the current state after the first $t_{1}+\ldots+t_{j}$ steps.

Obviously, $t_{j} \geq 0$, moreover some $t_{j}$ may be zero. For instance (the reader may check as an exercise), if $f>1$, then $t_{2}=0$. For the last group, we have $j=q^{f-1} \Delta-p^{f-1}+1=: N$. 
Notice that $v_{k_{j}}(x) \leq z_{j+1}$ for any $x \in V$. Therefore, by Lemma 2.1, we have

$$
\frac{v\left(k_{j}\right)}{z_{j+1}} \leq \frac{1-\lambda^{f}}{1-\lambda} \nu^{*}(\mathcal{F}) \text {. }
$$

Clearly,

$$
v\left(k_{j}\right)=\sum_{i=j+1}^{N} t_{i} z_{i}, \quad \text { for } 0 \leq j \leq N-1 .
$$

In total, we choose $t_{1}+t_{2}+\cdots+t_{N}$ vertices (that is the cardinality of $A$ with multiplicity), and they form an $f$-fold transversal of $(X, \mathcal{F})$. Thus, by (66) and (5), we obtain

$$
\begin{gathered}
\tau_{f} \leq t_{1}+t_{2}+\cdots+t_{N}= \\
=\left(\frac{v\left(k_{0}\right)}{z_{1}}+\sum_{j=1}^{N-1} v\left(k_{j}\right)\left(\frac{1}{z_{j+1}}-\frac{1}{z_{j}}\right)\right)=\frac{v\left(k_{0}\right)}{z_{1}}+\sum_{j=1}^{N-1} \frac{v\left(k_{j}\right) q^{1-f}}{z_{j+1} z_{j}} \leq \\
\leq \frac{1-\lambda^{f}}{1-\lambda} \nu^{*}(\mathcal{F})\left(1+\sum_{j=1}^{N-1} \frac{q^{1-f}}{z_{j}}\right)=\frac{1-\lambda^{f}}{1-\lambda} \tau^{*}(\mathcal{F})\left(1+\sum_{k=p^{f-1}+1}^{q^{f-1} \Delta} \frac{1}{k}\right) \leq \\
\leq \frac{1-\lambda^{f}}{1-\lambda} \tau^{*}(\mathcal{F})(1+\ln \Delta-(f-1) \ln \lambda),
\end{gathered}
$$

which completes the proof of Theorem 1.1.

2.5. Proof of Corollary 1.3. An $f$-fold transversal $A \subset X$ ( $A$ is a multiset) easily yields a fractional transversal: one sets the weight $w(x)=\frac{|\{x: x \in A\}|}{f}$ (cardinality counted with multiplicity) for any vertex $x \in X$. The total weight that we get from our construction in Theorem 1.1 is then

$$
\tau^{*}(\mathcal{F}) \leq \sum_{x: x \in V} w(x) \leq \tau^{*}(\mathcal{F}) \frac{1+\ln \Delta-(f-1) \ln \lambda}{f(1-\lambda)} \leq \tau^{*}(\mathcal{F})(1+\varepsilon) .
$$

2.6. Running time. Let $n$ denote the number of vertices and $m$ be the number of edges of the hypergraph. The adjacency matrix and $f$ are the inputs of the algorithm. As preprocessing, for each vertex, we create a list of edges that contain it (at most $\Delta$ ), which takes $n m$ operations. We keep track of the current state in an array $k$ of length $m$.

At each step, the following operations are performed. Computing the value of a vertex takes the addition of at most $\Delta$ numbers. Thus, finding the vertex of maximal value is $n \Delta$ operations. Picking that vertex means decreasing at most $\Delta$ entries of the array $k$ by one. We make at most $\frac{1-\lambda^{f}}{1-\lambda} \tau^{*}(\mathcal{F})(1+\ln \Delta-(f-1) \ln \lambda)$ steps.

With the $\lambda=0.287643$ substitution, in total, the number of operations is at most

$$
n m+O\left(\tau^{*} \max \{\ln \Delta, f\} \cdot \Delta n\right) \leq O(\max \{\ln \Delta, f\} \cdot \Delta n m) .
$$

\section{Multiple covering of SPACE - Proof of Theorem 1.4}

We denote by $K \sim T:=\left\{x \in \mathbb{R}^{d}: T+x \subseteq K\right\}$ the Minkowski difference of two sets $K$ and $T$ in $\mathbb{R}^{d}$. For $K, L \subset \mathbb{R}^{d}$, and $f \geq 1$ integer, we denote the $f$-fold translative covering number of $L$ by $K$, that is, the minimum number of translates of $K$ such that each point of $L$ is contained in at least $f$, by $N_{f}(L, K)$. We denote the fractional covering number of $L$ by $K$ by $N^{*}(L, K):=\tau^{*}(\mathcal{F})$, where $\mathcal{F}:=\{x-K: x \in L\}$ is a hypergraph with base set $\mathbb{R}^{d}$, see details in [Nas14], or [AAS15]. 
Theorem 3.1. Let $K, L$ and $T$ be bounded Borel measurable sets in $\mathbb{R}^{d}$ and let $\Lambda \subset \mathbb{R}^{d}$ be a finite set with $L \subseteq \Lambda+T$. Then

(7)

$$
N_{f}(L, K) \leq\left\lceil 3.153 N^{*}(L-T, K \sim T) \max \left\{\ln \left(\max _{x \in L-K}|(x+(K \sim T)) \cap \Lambda|\right), f\right\}\right\rceil .
$$

If $\Lambda \subset L$, then we have

$$
N_{f}(L, K) \leq\left\lceil 3.153 N^{*}(L, K \sim T) \max \left\{\ln \left(\max _{x \in L-K}|(x+(K \sim T)) \cap \Lambda|\right), f\right\}\right\rceil .
$$

Theorem 3.1 is the $f$-fold analogue of [Nas14, Theorem 1.2], where the case $f=1$ is considered. For completeness, we give an outline the proof.

Proof of Theorem 3.1. To prove (7), consider the hypergraph with base set $\mathbb{R}^{d}$ and hyperedges of the form $u-(K \sim T)$, where $u \in \Lambda$. An $f$-fold transversal of this hypergraph clearly yields an $f$-fold covering of $L$ by translates of $K$. A substitution into (3) yields the desired bound. We omit the proof of (8), which is very similar.

Using this result, one may prove Theorem 1.4 following [Nas14, proof of Theorem 2.1], which is the proof of Rogers' density bound (4). We give an outline of this proof.

Proof of Theorem 1.4. Let $C$ denote the cube $C=[-a, a]^{d}$, where $a>0$ is large. Our goal is to cover $C$ by translates of $K$ economically. We only consider the case when $K=-K$, as treating the general case would add only minor technicalities.

Let $\delta>0$ be fixed (to be chosen later) and let $\Lambda \subset \mathbb{R}^{d}$ be a finite set such that $\Lambda+\frac{\delta}{2} K$ is a saturated (ie. maximal) packing of $\frac{\delta}{2} K$ in $C-\frac{\delta}{2} K$. By the maximality of the packing, we have that $\Lambda+\delta K \supseteq C$. By considering volume, for any $x \in \mathbb{R}^{d}$ we have

$$
|\Lambda \cap(x+(1-\delta) K)| \leq \frac{\operatorname{vol}\left((1-\delta) K+\frac{\delta}{2} K\right)}{\operatorname{vol}\left(\frac{\delta}{2} K\right)} \leq\left(\frac{2}{\delta}\right)^{d} .
$$

Let $\varepsilon>0$ be fixed. Clearly, if $a$ is sufficiently large, then

$$
N^{*}(C-\delta K,(1-\delta) K) \leq(1+\varepsilon) \frac{\operatorname{vol}(C)}{(1-\delta)^{d} \operatorname{vol}(K)}
$$

By (7), (9) and (10) we have

$$
N_{f}(C, K) \leq\left\lceil 3.153 \frac{1+\varepsilon}{(1-\delta)^{d}} \frac{\operatorname{vol}(C)}{\operatorname{vol}(K)} \max \left\{d \ln \left(\frac{2}{\delta}\right), f\right\}\right\rceil .
$$

Thus, we obtain an $f$-fold covering of $C$. We repeat this covering periodically for all translates of $C$ in a tiling of $\mathbb{R}^{d}$ by translates of $C$, which yields an $f$-fold covering of $\mathbb{R}^{d}$. The density of this covering is at most

$$
N_{f}(C, K) \operatorname{vol}(K) / \operatorname{vol}(C) \leq\left\lceil 3.153 \frac{1+\varepsilon}{(1-\delta)^{d}} \max \left\{d \ln \left(\frac{2}{\delta}\right), f\right\}\right] .
$$

We choose $\delta=\frac{2}{d \ln d}$, and a standard computation yields the desired result.

Acknowledgement. We thank the referees, whose comments helped greatly to improve the presentation. 


\section{REFERENCES}

[AAS15] S. Artstein-Avidan and B. A. Slomka, On weighted covering numbers and the Levi-Hadwiger conjecture, Israel Journal of Mathematics 209 (2015), no. 1, 125-155.

[BDS04] P. Berman, B. DasGupta, and E. Sontag, Randomized Approximation Algorithms for Set Multicover Problems with Applications to Reverse Engineering of Protein and Gene Networks, Approximation, Randomization, and Combinatorial Optimization. Algorithms and Techniques: 7th International Workshop on Approximation Algorithms for Combinatorial Optimization Problems, APPROX 2004, and 8th International Workshop on Randomization and Computation, RANDOM 2004, Cambridge, MA, USA, August 22-24, 2004. Proceedings, 2004, pp. $39-$ 50 .

[Dob82] G. Dobson, Worst-Case Analysis of Greedy Heuristics for Integer Programming with Nonnegative Data, Mathematics of Operations Research 7 (1982), no. 4, 515-531.

[FK06] T. Fujito and H. Kurahashi, A Better-Than-Greedy Algorithm for $k$-Set Multicover, Approximation and Online Algorithms: Third International Workshop, WAOA 2005, Palma de Mallorca, Spain, October 6-7, 2005, Revised Papers, 2006, pp. 176-189.

[FNN16] N. Frankl, J. Nagy, and M. Naszódi, Coverings: variations on a result of Rogers and on the Epsilon-net theorem of Haussler and Welzl, arXiv:1607.02888 [math.MG] (2016).

[FT04] G. Fejes Tóth, Handbook of discrete and computational geometry (2nd edition), 2004, pp. 25-53.

[FT76] G. Fejes Tóth, Multiple packing and covering of the plane with circles, Acta Math. Acad. Sci. Hungar. 27 (1976), no. 1-2, 135-140. MR0417930

[FT79] G. Fejes Tóth, Multiple packing and covering of spheres, Acta Math. Acad. Sci. Hungar. 34 (1979), no. 1-2, 165-176. MR546731

[Für88] Z. Füredi, Matchings and covers in hypergraphs, Graphs Combin. 4 (1988), no. 2, 115-206.

[Joh74] D. S. Johnson, Approximation algorithms for combinatorial problems, Journal of Computer and System Sciences 9 (1974), no. 3, $256-278$.

[Lov75] L. Lovász, On the ratio of optimal integral and fractional covers, Discrete Math. 13 (1975), no. $4,383-390$.

[Nas14] M. Naszódi, On some covering problems in geometry, arXiv:1404.1691 [math] (April 2014). To appear in Proc. AMS.

[PA95] J. Pach and P. K. Agarwal, Combinatorial geometry., New York, NY: John Wiley \& Sons, 1995 (English).

[Rog57] C. A. Rogers, A note on coverings, Mathematika 4 (1957), 1-6.

[RV98] S. Rajagopalan and V. V. Vazirani, Primal-dual rnc approximation algorithms for set cover and covering integer programs, SIAM Journal on Computing 28 (1998), no. 2, 525-540, available at http://dx.doi.org/10.1137/S0097539793260763.

[Ste74] S. K Stein, Two combinatorial covering theorems, Journal of Combinatorial Theory, Series A 16 (1974), no. 3, $391-397$.

[Vaz01] V. V. Vazirani, Approximation algorithms, Springer-Verlag New York, Inc., New York, NY, USA, 2001.

(M. Naszódi) Elte, Dept. of Geometry, Lorand Eötvös University, PÁzmány Péter Sétány 1/C Budapest, Hungary 1117

(A. Polyanskii) Moscow Institute of Physics and Technology, Technion, Institute for InFORMATION TRANSMISSION PROBLEMS RAS.

E-mail address: marton.naszodi@math.elte.hu and alexander.polyanskii@yandex.ru 